\section{ON CERTAIN CASES IN WHICH THE FORAMEN OVALE WAS STILL PATENT IN THE ADULT.}

By JohN W. OGLE, M.B.Oxon., F.R.C.P., Assistant-Physician to St. George's Hospital.

IN the year 1851, whilst curator of the Pathological Museum at St. George's Hospital, I took the opportunity of examining above one hundred and forty human hearts taken indiscrimin ately after death, for the purpose of ascertaining the condition of their muscular fibres.* Of this number, I examined sixtytwo with a view of discovering the condition also of the foramen ovale, and found that out of the sixty-two, there were thirteen hearts which retained this foramen yet incompletely closed, although to a variable degree; thus giving a proportion of about one in five hearts in which communication of some kind or other existed between the cavities of the two auricles. This is, I believe, a greater proportion than is generally considered to exist in the healthy human adult heart.

These particulars, observed at the period I mentioned, have hitherto not been made available by me; but I have retained them in my note-book, thinking that at a future time some opportunity for their use might occur. Such an opportunity has been offered, in consequence of the observations of $\mathrm{my}$ friend Dr. Markham, regarding a case published by him in the number of this Journal for April 4th, 1857. In this case of Dr. Markham's, a loud murmur coincident with the systole of the ventricles, and therefore synchronous with the diastole of the auricles, was heard along the base of the heart and in the entire left subclavicular region. Of some of his remarks in connexion with the case, I shall speak at greater length further on.

Having mentioned the fact of the comparatively frequent occurrence of an incomplete closure of the foramen ovale, I must qualify the observation by remarking, that the patency noticed allowed of very different degrees of communication between the two sides of the heart. In some of the cases, the opening remaining in the septum of the auricles was a mer fissure or oblique slit, arising simply from want of such an adhesion between the valves and the margins of the isthmus, as is wont to exist after the completion of the drawing up of the valvular fold and other preliminary contractile actions of neighbouring parts. This defective adhesion, I may remark, is thought by Dr. Peacock to be the result of unequal pressure in the auricles from the blood, owing to the slow establishment of the pulmonary circulation after birth. The oblique slits, to which I have alluded, were directed variously, as it were, opening in some cases more widely from above, and in others from below, and would have allowed of the blood passing, as it would seem, more freely in some cases from the left into the right auricle, but in the majority of cases from the right into the left auricle. In several of these cases, the communicating opening was not merely that of a chink or slit, but was a decided round or oval aperture; in one case, admitting of the entrance of the tip of the little finger. In one or two cases, the opening was of a reticular character, having the appearance as if it had been produced by a falling short of one part of the margin of the valvular fold, so that it failed to be raised to the level of the isthmus, to which it ought to have been adherent; and as if the deficiency had been in part compensated for by bands passing across the vacancy. I was careful in my search for any trace of ulceration or of rupture which might have accounted for the opening, but this did not in any case exist.

I have lately referred to the life histories of those cases which $I$ have mentioned as presenting an imperfect closure of the foramen ovale, in order to ascertain what physical signs were noticed before death, which might be attributed to this imperfect closure.

I will now enumerate the cases, and describe briefly the condition of the heart and also of the lungs in each of the thirteen cases in which I found the foramen only partially closed. Afterwards, I will add a short statement of the stethoscopic signs as observed during life, whenever I find that they were noted by my friend and colleague Dr. Barclay, who at that time was medical registrar at St. George's Hospital.

CASE I. A patient, aged 41 , affected by pneumonia, rheumatism, and eventually pericarditis. The chest was carefully stethoscoped when the patient came into the hospital, and special search was made for signs of peri- or endocarditis, but

* The results of the examination for this purpose are detailed at page 68 of the 4th volume of the "'Transactions of the Pathological Society". no bruit or friction-sound whatever was heard. After a time, when the anticipated pericarditis had appeared and become established, the ordinary murmurs were heard.

CASE II. A patient, aged 37, was admitted into the hospital, and died with pulmonary phthisis and vomicæ. After death; the heart, which weighed eight and a half ounces, was found healthy and natural, excepting the patency of the oval foramen. In the life history it is expressly observed that " the only thing made out by physical exploration of the chest was slight harshness of breathing on the right side of the chest." These particulars are given, but there is no mention whatever of any unnatural sounds about the heart.

CASE IIr. A patient, aged 25, was admitted into the hospital with dropsy and other symptoms of diseased kidneys. There were also symptoms of congestion of the lungs and enlarged heart; and a regurgitant murmur was heard at the root of the aorta. In the life history it is expressly stated that there was. no double murmur; in other words, that no murmur existed synchronous with the systole of the ventricles. After death, the heart, which weighed twenty ounces, showed small fibrinous granulations upon the surface of the aortic valve-flaps.

CASE IV. A patient, aged 38, died in St. George's Hospital of phthisis with vomicæ. The heart, which weighed ten ounces, was healthy, excepting the open foramen. In the life history: a careful description of the physical exploration of the chest is given; but there exists no mention of any unnatural heart. sounds.

CaSE v. A patient, aged 32, who died of acute pleurisy and congestion of the lungs. In the life history, the physical signs, which were observed as connected with the lungs, are given; but no mention is made of any murmurs about the heart.

CASE vr. A patient, aged 37, who died of abscess within the brain, connected with disease of the inner ear. After death, the lungs were found to be greatly gorged with blood. The heart, which weighed nine and a quarter ounces, presented small shreds and granulations of recent fibrine attached to the aortic valve-flaps; but otherwise, saving the patent foramen, it was natural. In the life history, it is stated that "the lungs appeared only to be affected with bronchitis."

CASE vir. A patient, who died with diseased kidneys and hypertrophy of the heart. After death, the lungs were found to be very gorged with blood. The heart weighed twenty-one ounces. Its walls were thick, and its cavities dilated; and its aortic valve-flaps, which were only two in number, were rigid and unpliable. It is expressly stated in the life history that "the heart's sounds were very feeble, but nothing abnormal was found."

CASE virr. A patient, aged 32, who died of pleurisy, and was moribund when brought into the hospital. Slight scrofulous deposit was found within the lungs after death; and one lung was rendered quite useless by compression, from fluid accumulated within the pleural cavity. The heart weighed fourteen ounces, its walls being thickened.

CASE IX. A person, aged 16, who died of peritonitis with bronchitis and pneumonia. The heart was natural, excepting the patent foramen. In the life history, the sounds of the heart are not alluded to directly or indirectly.

CASE X. A patient, aged 38, was poisoned by prussic acid, and brought into the hospital dead. Excepting the open foramen, the heart was found to be natural.

CASE XI. A patient, aged 60, was brought into St. George's Hospital with disease of the bones of the foot, and died of pulmonary phthisis with vomicæ. The heart was soft and flaccid; but, excepting the open foramen, was quite natural. In the life history, I find nothing whatever bearing upon the sounds of the heart.

CASE XII. A patient, aged 45, who died of peritonitis and pneumonia. The heart, which weighed only five and a half ounces, was small; but, excepting the open foramen, natural. In the life history, I find nothing bearing upon the sounds of the heart.

CASE XIII. A patient, aged 48, was admitted with a fistula in ano, and died of pulmonary phthisis. The heart, which weighed nine and a half ounces, was soft and lacerable; the walls of the right ventricle being thinner than usual. In the life history, I find nothing relating to the sounds of the heart.

Thus, in the preceding cases, thirteen in number, in which the foramen ovale was found to be but incompletely closed after death, there are seven in whose histories it is emphatically stated or to be inferred that no murmur synchronous with the systole of the ventricles, that is also synchronous with the diastole of the auricles, existed; and only one in which there 
is notice of any murmur with the diastole of the ventricles, that is, with the systole of the auricles. Consequently, excepting in this last single instance, we have the fact of six hearts in which more or less patency of the foramen ovale existed, but did not give origin to any unnatural sound whatever about the heart. In the solitary instance (Case III), in which a regurgitant or diastolic murmur (so-called) was heard, the heart was very large and soft, and recent granulations and shreds of fibrinous material, deposited from the blood, were adherent to the aortic valve flaps. It may therefore well be believed that this murmur was seated in the left ventricle, and had nothing to do with the septum of the auricles; if it had any such connection it must have been produced during the systole of the auricles, and therefore not at all similar to that in Dr. Markham's case which took place during the ventricular systole, and was supposed by him to be produced during the diastole of the auricles. As regards the last six cases, in which the closure of the foramen ovale was imperfect, I am sorry to say that the life history does not afford data from which any conclusive evidence as to the presence of cardiac bruits may be derived. It will be remembered that in all these thirteen cases the patients were adults. In none of them was there any cyanosis noticed during life, or congenital malformation of the heart or its vessels found after death. Moreover, there was no such condition of the valves or orifices of the heart as should primarily or secondarily tend in any material degree to impede the blood's exit from the auricles, and thus, as it were, throw greater pressure and stress upon the septum along with other parts of their walls, and lead to a mixture of the venous and arterial blood through the foramen ovale. There was, how. ever, in many cases marked and extensive disease of the lungs, such as their occupation by the products of inflammation or scrofulous deposit, which, though to a less extent, would have a similar tendency as regards the right auricle.

I may be permitted here, as I have his sanction, to append a case lately brought before the notice of the Pathological Society by Dr. Ogier Ward, which I will curtail. It bears strongly upon the point I am attempting to illustrate.

The case was that of a child who died eleven days after birth, having been cyanotic and affected with dysphagia and choking on swallowing. The lungs and heart were auscultated during life, and it was noticed that the heart's sounds were natural, no bruit existing. After death, congenital communication between the trachea and œsophagus was found, with enlargement of the pulmonary artery and patency of both the ductus arteriosus and foramen ovale. The preparation is now in the museum of the College of Surgeons. This case, added to those related above, makes the seventh case in which the foramen ovale was more or less patent during life, and in which we have evidence that no bruit was thereby produced.

Having epitomised as briefly as possible all the cases connected with the subject of which I have any accurate note, I will say a few words regarding Dr. Markham's interesting observations.

In the case related by him, which I before noticed, nothing unnatural was found after death in the condition of the heart beyond a patent state of the foramen ovale allowing the blood to pass readily from the right to the left auricle, but not permitting it to pass in the opposite direction, excepting through two narrow slits. Among other remarks in connection with it, Dr. Markham suggests the very important query whether this peculiar state of the foramen was sufficient to account for the very marked and widely extended systolic murmur heard during life; so marked, indeed, that before death it was described as loud, rough, and prolonged, audible over the whole præcordial region and over the upper part and along the right border of the sternum, and in the whole of the upper half of the interscapular space. Dr. Markham supposes that in many cases of open foramen ovale under certain circum stances this state induces murmur during the systole of the heart. If this state of the foramen be accompanied by other unnatural states of the heart producing any murmur, then the murmur caused at the septum of the auricles becomes masked and hidden; and if there be no such disease in other parts of the heart, then no heart symptoms arise, and thus the heart remains unexamined during life, and consequently the aforesaid auricular murmur not detected. He says, "Is it not possible that such a murmur may have been present in many cases simply of open foramen ovale, but was not discovered during life because the disturbance to the circulation produced by the same lesion were not of themselves of a nature marked enough to excite attention." Again: "Cases where the foramen ovale must have been partially open during life, and 501 yet where no symptoms to mark the fact had been observed are frequently met with; and assuredly in the majority of these cases it would be a complete begging the question to affirm that no murmur really existed during life. It is more correct to say that in the very great majority of cases of simple open foramen ovale, disturbances in the circulation do not occur, and therefore no examination of the heart is made during life. Practically speaking, how many cases are there on record where simple foramen ovale has been found after death, in which the observer can affirm that no murmur existed during life? I suspect very few." It is with this last question only of Dr. Markham's that I am now concerned; and though in what I have brought forward I do not say that I have shown reason for supposing that a murmur cannot, under any circumstances, be produced by the passage of blood through an open foramen ovale, yet I have, as I think, shown that there are numbers of cases under ordinary circumstances in which, although facility for the passage of blood from one auricle to another exists, yet no unnatural sound is thereby of necessity produced. This is so, judging from the cases adduced, even when considerable obstruction to the blood's passage through the lungs must in many cases have been present. It may be that with a certain degree of patency, a certain tension of the boundaries of the foramen, a certain quality of the blond as to consistence, and a determinate force with which the blood is propelled by one or other auricle; under all these conditions it may be a murmur might possibly be caused at an open foramen ovale. More extended observations than have hitherto been made on the matter are, however, yet required to establish the fact.

I will not here enter into the question of the greater probility of the blood's passing from one chamber to anotker through an open foramen ovale during the systole rather than during the diastole of the auricles, a point mooted by Dr. Markham, or vice versi ; or whether, again, as Dr. Hope and others have thought, the auricles are always full, a fact which I doubt, but which, if true, would I think seem to show that the circumstances of filling or emptying the auricle during systole or diastole, would be of no consequence in the production of a murmur in cases where the foramen is not fully closed, inasmuch as the septum and the valve would then always be on the stretch, and consequently always in the same invariable state of vibration. Nor will I enter into the question of the abstract possibility or otherwise of vibrations being produced at the margins of an open foramen ovale, or of the valve partially closing it, which might give rise to a murmur. These points and many others, such as the general anatomy and physiology of the foramen ovale, its method of closure, and the hinderances to its closure, might well here be discussed in furtherance of our entire knowledge of the subject in hand; but I have not entered upon them as they do not fall within the character and limits of the immediate question which I undertook in some degree to answer; viz., "How many cases are on record where simple open foramen ovale has been found after death, in which the observer can affirm that no murmur existed during life?"

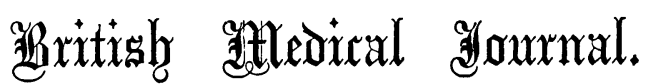

$$
\text { SATURDAY, JUNE 13тн, } 1857 .
$$

\section{THE MEDICAL REFORM BILLS.}

IT was fully expected that the discussion on Mr. Headlam's Medical Bill would take place on Tuesday last. It is reported that Lord Elcho intends to withdraw his notice of motion, "That the Bill be read that day six months," and that the discussion will take place upon its merits. We are inclined to think that such will be found to be the case if it comes on to-night (Thursday), as it most probably will. We call attention to the very important deputation, consisting of delegates from nearly every College of Medicine, and from most of the Universities in the three kingdoms, which waited upon the Prime Minister, at his private residence, on Tues- 